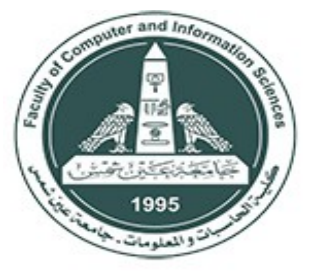
International Joumal of Intelligent Computing and Information Science

\title{
AN INTELLIGENT SYNCHRONOUS E-LEARNING MANAGEMENT SYSTEM BASED ON MULTI-AGENTS OF LINKED DATA, ONTOLOGY, AND SEMANTIC SERVICE.
}

\author{
Amin, Ahmed E* \\ Associate Professor \\ ahmedel_sayed@mans.edu.eg
}

\begin{abstract}
ahmedel_sayed@mans.edu.eg
Abstract: In the definition of the term e-learning, the researchers considered that the use of electronic media as a means of distance learning is in a way that enables both instructors and learners to interact through content on the web. So, e-learning in higher education worldwide has an important role in the activities of teaching and learning. Web technology for the construction of synchronous e-learning has been successfully developed to provide automatic educational content. The most important developments in this area are the semantic web that provides distributed information with a specific meaning precisely so that e-learning becomes an efficient relevant task that grows in a new dynamically changing learning requirements and needs of the learner. This paper has contributed to this goal by a system known as Intelligent Synchronous E-Learning Management System (ISEMS) that based on multi-agent such as semantic service, ontology, and linked data. Each agent represents the basis for organizing content resources, sequence learning, and adaptation. ISEMS hypertext structures from distributed metadata are utilized from the semantic web resource description formats. The description is based primarily on the content, learning material content's structure, and context so then provides adaptable and personalized access to these materials objects.
\end{abstract}

Keywords: Intelligent E-Learning Management System, Multi-Agents, Ontology, Semantic Service Web, Linked Data.

1. Introduction:

In recent years, new terminology has emerged as a result of the internet widespread use such as Infobestity [1], Infoxication [2] and Information Overload [3]. All of these terms lead to difficulties in understanding issues and problems and making effective decisions when one is overwhelmed with a lot of information about this issue [4]. 
The information overload can be classified into three factors [5]: cognitive, affective, and psychomotor as shown in Figure1. The cognitive factors include lack in both prior subject knowledge, technical skills, and English proficiency additionally the complexity of the tasks.

Fig. 1. Classification of Information Overload

The information overload is affecting is overwhelmed with a lot of many fields, including education. In education; the information overload phenomenon is defined as "the point at which students" working memory capacity is exceeded, and excessive information and stimuli from E-learning environment interfere with their learning" [6].

The ability to rapidly produce and disseminate information on a wider scale because of the advances of information technology (IT) has been a major cause of increased overload of information [7]. There are two major drawbacks in educational systems that are based on information technology: first, the provider which offer the course content cannot suit individual students' learning ability. Second, the low transfer speed of the massive multimedia courses content. Thus, intelligent learning systems (ILS) should be able to distinguish students according to their individual differences, and develop a teaching strategy that increases both; the student's performance and the learning efficiency, especially when providing the basic requirements of ILS as plenty learning material, efficient resources searching ability, and interactive interface.

Thus, finding the right information that users need from the web that matches search terms, form and theme, poses a major challenge in education [8]. The intelligent learning system offers a solution to the e-learning weakness and challenges.

In response to the challenge of information overload, an Intelligent Synchronous E-Learning Management System (ISEMS) accompanied by the concepts of semantic services, e-learning ontology, and linked data is proposed and applied to the establishment of e-learning management.

Current E-learning systems which used web 2.0 are focused not only on courses content delivery but also on personalized and adaptive learning style of learners. These systems suffer while read and write handling in Web 2.0, so they are facing some challenges to meet the requirements of the semantic web. There are some challenges facing the existing e-Learning systems, including managing the huge e-Learning content that is continuously grows on the web, meeting the learner's requirements when searching for an electronic learning content, representing the knowledge in a format that is easy to read and makes it capable of thinking and thus allowing the re-use of e-Learning objects.

There are three different techniques that are used in ISEMS to address information overload challenges namely Semantic Services, Linked Data, and Ontology Metadata. Each technique focuses primarily on a particular set of tasks.

One of the reasons for the use of semantic services in the proposed system is the integration of content ontologies with a keyword-based search for semantic search to meet the needs of users which considered a new technology in information retrieval.

Ontology, in the previous decade, is defined by different meanings in different applications [10]. One of the most important definitions is "The shared concepts have explicit and formal specifications" [11], so there are many ontology characteristics like formality, explicitness, being shared, conceptuality, and domain specificity which considered as the specification of domain knowledge. Therefore, in the proposed system (IEMS), an ontology identifies the semantics of terms and the domain data meanings in a formal and explicit 
manner, thereby providing a shared understanding in IEMS to support communication among learners and applications.

Ontology is characterized by the ability to support the sharing and reuse of formally represented knowledge by explicitly articulating concepts, relationships and axioms when defining the course specifications of an IEMS. Therefore, ontologies will be applied in the proposed system to knowledge modeling for information sharing among applications, and to boost knowledge reuse.

Linked data [12] belong to the ways of publishing and linking data on the web so then applied to the data extracted from the educational sites. The semantic research community [13] has benefited from the possibilities offered by the integration of semantics into research systems, their ability to support advanced research experiences, and the resolution of complex information needs.

The outline of the paper is divided into six sections, section 1 presents information about the components of the proposed system. Intelligent E-learning systems are presented in Section 2. Section 3 provides basic concepts of Ontology, linked data and the semantic web. Section 4 Proposed system. Section 5 shows experimental and results and concluding remarks are introduced in Section 6.

\section{Intelligent E-Learning Systems:}

Intelligent E-Learning Systems (IELSs) are an important advance development in computer-based learning systems, in which the tutoring strategies are adapted to the requirements and abilities of learners and expertise of instructors. For the success of any IELSs, learner requirements should be taken into consideration when retrieving relevant learning materials. Figure 2 shows, the main modules of IELSs are the Expert knowledge model, Student model, Tutorial model, and User Interface model.

Fig. 2: General Intelligent E-Learning System Architecture.

To IELS be a more effective system to meet the learner's requirements has been developed by the addition of multi-agent to retrieve information in order to provide resources for learning and reuse such as ontology, web semantic and linked data.

\section{Multi-agent-Based E-Learning Management System:}

There are several reasons [14] that lead us to the development of e-learning management systems to improve the static sets of services and overcome on the time-consuming maintenance requirements of e-learning courses, and also support students and meet their needs and take into account their backgrounds and skills. Decisions of what content will be delivered next to users are the important aim of e-learning system which is achieved by the evaluation of user's behavior.

An e-learning strategy does not depend only on content transfer, but present to users the best-suited material, administrative purposes and managing the user's performance and learning environment by tracking and evaluating his behavior. Both of learner and supervisor use e-learning system.

Intelligent E-learning systems (IEs) take into account in their strategy not only the simple transmission of content but also the learner's communication with his supervisor, colleagues, and the surrounding environment which is represented in the management system. In order to use IEs to provide materials that suit the learner in terms of previous experience and to meet his needs, it must be taken into consideration the author, the content and how to manage it. This paper proposes the development of intelligent education systems using intelligent agencies based on the web semantic services and ontology and linked data to assist in content creation and management such as making specialized material, assignments, quizzes, etc.

\subsection{Semantic Services Agent-based E-Learning System:}


Visions of Semantic Services (SS) are using semantic structures to improve the results of exploration on the web and also can be used techniques on the web to mining Semantic Web itself [15]. The wording SS emphasizes this possible interaction between both web mining and web services. So it's possible to use web logs for any courses available on any e-learning system for investigation of semantic information.

Semantic Services Agent (SSA) can be constructed from ontologies, agents, and reasoning systems. This techniques, and their associated technologies, can be effectively combined in a Service-Oriented Architecture (SOA) [16]. The SOA is a software architecture style based on distributed components that have been dynamically created, can be called services that deal with three major activities; which are the remote invocation of services, the discovery of services that match certain criteria, and the composition of services to form applications.

The SSA consists of two main parts; [17] are Model Reference (MR) referring to semantic concepts and Schema Mappings (SM) that determine the data transformations between the Extensible Markup Language (XML) data structure of messages and the associated semantic model. To refer to one or several semantic concepts, we use the MR, which is an extension attribute that can be applied to any element of the XML schema. Whereas, SM is represented by two attributes known as lifting mappings and lowering mapping. They both work in reverse, where the first, converts XML data from a Web service message into a semantic model (for instance, into RDF data that follows some specific ontology) and the other converts data from a semantic model into an XML message.

The semantic service structure (SS) [18] consists of the required functions associated with the user's needs to understand the technical situation in a particular field of science. Thus the user's query can be understood and answered by summarizing and synthesizing several different aspects such as the most relevant parts related to a specific area of a document, the characterization of research initiatives and leading scientific groups, the characterization of open problems and the most significant concepts, historical trends of progress in similar domains etc. Therefore, the framework needs to be implemented combining the ontologies and the standard search capabilities based on the keywords, dictionaries, and glossaries $[19,20]$ as well as reasoning and hierarchical knowledge representation [21, 22], based on domain knowledge acquired from experts and users as shown in figure 3.

Fig. 3: The Semantic Service Agent Architecture.

\subsection{Ontology Agent-based E-Learning System:}

There are two main types of ontologies, light-weight that involve class hierarchy (taxonomy) e.g. classes, subclasses, attributes and values and heavy-weight that include axioms and constraints [23]. Ontology Agent (OA) methodologies [24, 25] is support ontology building for centralized ontology in e-learning application and focus on the consensus building process in collaborative ontology engineering. OA methodology divides the ontology building process in a varying number of stages such pre-development, ontology development, and post development. Each stage contains a number of activities as shown in figure 4.

Fig. 4: Ontology Engineering Activities.

To name a few, the importance of any particular activity within a methodology primarily depends on the SS characteristics, Domain, and curriculum application based on ontology, the complexity of the ontology to be built, the information sources available, the ontology engineers experience. 
The OA management activities [26] are responsible for the organizational preparation of the procedures carried out. In the pre-development phase, examines the use of an ontology in a Semantic Service, the domain, the curriculum, and pedagogical resources. Domain analysis, conceptualization, and implementation are light-weight ontology development activities (LwODA). In parallel with LwODA, there are performed ontology support activities such knowledge acquisition (KA), evaluation, reuse, and documentation. The maintenance and the use of the ontology are post-development activities.

\subsection{Linked Data Agent-based E-Learning System:}

Linked Data (LD) is a term means using semantic queries to publish data that is easily interlinked and managed over the Internet. Thus facilitating the determination and interlinking of data sets which leads to exchanged, reused and integrated [27].

Fig. 5: Linked Data Agent Architecture.

The aim of the Linked Data Agent (LDA) [28] is to identify datasets that are available under open licenses, re-publish these in Resource Description Framework (RDF) on the Web and interlink them with each other. LDA adopts a set of technical rules for publishing data on learning web pages that have become represents as Linked Data Layers [29] as shown in figure 5:

1. Logic Application Layer (LAL) manage two types of linked data resources the first type are the RDF sources, and the second are the HTML files such as; images, other binary files ... etc. Both resources might contain a description of a number of different concepts such as a specific domain entity; Status, Friendship, Product, Order, Bug, etc. All description-resources files are aggregated in containers that are known as Linked Data Container (LDC).

2. Data Virtualization Layer (DVL), in this layer, the linked data users are responsible for processing the users linked data in order to produce new linked data. The DVL is divided into two layers the first layer is Transformation Layer (TL) and the other is Conceptual Layer (CL). TL, separates the distinct linked data with RDF format, whereas the other formats from linked data are encapsulated in the form of cartridges similar to RDF format. On top of TL there are storages to store both Linked data formats. In CL, Is responsible for reusing all databases of linked data to reuse as part of a service-oriented architecture that focused on reusing middleware infrastructure for building personal page application based on the needs of the user.

3. Search Layer (SL): The query language (SPARQL) is used in the Linked data agent to obtain the relevant data. The obtained data is driven by query URIs data sources and partial results.

\section{Intelligent E-Learning System based Multi-Agent:}

An advocated approach to design an intelligent e-learning system based on multi-agent $\left(I E_{L} S M_{A}\right)$ is constitutes of: (a) Intelligent Learning Environments, (b) Semantic Service Agent, (c) Ontology Agent, and (d) Linked Data Agent as shown in figure 6. The core of the proposed system is the personalized instructions which is represented as the needs of learners, the vision of instructors, and views of educational experts to design the e-learning outline architecture.

An $I E_{L} S M_{A}$ include notions and requirements for collect information and terms resulting from a user's query in different forms such as Meta-data, Syntactic, and Semantics. The ontology agent (OA) is used to transform the formal user's query to different forms of queries. The results are presented in object and concept forms by using SS, then the objects are arranged and linked with its relevant concepts by using LD. The OA can be used for understanding the meanings of terms and increasing flexibility in using different 
names for the same attribute or relation. Increasing flexibility is useful for the user interface and for integrating modules implemented by different system components.

Fig. 6: The Proposed System Architecture.

Web Ontology Language (OWL) [30] is used to implement the OA activities such as matching or measuring the similarity between different query forms and the results, answering queries and navigation and improving queries in the user interface. An $I E_{L} S M_{A}$ is structured as shown in figure 7 as Services Analysis, Searching Engine, and Domain Synthesis.

Fig. 7: Outline the $I E_{L} S M_{A}$ architecture.

\subsection{Services Analysis:}

The system contains information about important terms, concepts and objects. A term consists of strings representing a word or a phrase consisting of a few words which has been normalized using a stemming algorithm [31]. The Term Frequency - Inverse Document Frequency $(t f-i d f)$ [32] was used to measure the importance of a term $\left(t_{i}\right)$ in a text $\left(d_{j}\right)$ after the maximal number of words in a term was parameterized and chosen appropriately. The $t f-i d f$ technique is defined as $t f_{i, j} \times i d f_{i}$, where $t f_{i, j}$ refers to the frequency of $\left(t_{i}\right)$ in $\left(d_{j}\right)$, and $i d f_{i}$ refers to the general importance of term ti in the set of all documents of the system.

The information about important terms is collection TERMS of pairs $\left(t_{i}, i d f_{i}\right)$, then the threshold (h) for

$(i d f)$ is chosen appropriately, where $t f-i d f_{i, j} \geq h$. The $I E_{L} S M_{A}$ system is implemented to measures the similarity between a string and a term.

Meaning of the concept can be understood as an ID and list of label and weight pairs. The label is a term while the weight is a number whose value is enclosed between zero and one indicates the accuracy of the term has that meaning. The similarity between a term $(\mathrm{t})$ and a concept $(\mathrm{c})$ with ID is implemented.

The original form of a publication may be an image such as a document has been OCR'ed or hyperlinked. The objects are represent a URI and documents as a tree. Using the terminology of XML to represent the objects. An object is an element have attributes, each attribute has a name and a simple value. There are differences between Elements and attributes of XML, attributes are simple (i.e. without structure), functional, and their order are inessential, while the order of elements is essential [33].

\subsection{Search Engine:}

The user interface module (UIM) is used as a general form for user's queries, where the search engine does not provide this service. The user queries are preprocessed by UIM before is passing to the search module. The list of phrases is replaced by a set of weighted phrases that consists of concepts and objects in the preprocessing phase. The queries are combined as searching based on metadata, searching based on syntactic keywords and "semantic" searching:

$$
Q=\left(Q_{\text {Met }}, Q_{\text {Syn-Ph }}, Q_{\text {Sem-Ph }}, Q_{\text {Con }}, Q_{i-O b j}, Q_{W}\right)
$$

Where; $Q_{\text {Met }}$ is a logical formula over the metadata of the system with only one free variable standing for the queried object (like SPARQL [34]). $Q_{S y n-P h}$ is a set of pairs weighted phrases $\left(S t_{i}, W_{i}\right)$ where $S t_{i}$ is a string and $W_{i}$ is the corresponding weight for $S t_{i} . Q_{S e m-P h}$ is the same as $Q_{S y n-P h}$ except that the word "syntactically" is replaced by "semantically". $Q_{\text {Con }}$ is a set of weighted concepts (used to matching with queried objects). $Q_{i-O b j}$ is a set of weighted objects (used to "semantically" matching with 
queried objects w.r.t. similarity of topics/fields). $\quad Q_{W}$ is a function from $[0,1]$. The result of a query $\Phi$ is specified by:

- The function $\dot{i}_{S o}^{S y n}(x, y)$ represents the syntactic similarity, it maps a pair consisting of a string (x) and an external object $(y)$ to a real number in $[0,1]$.

- The function $i_{S o}^{\text {Sem }}(x, y)$ that represents the semantic similarity, it maps a pair consisting of a phrase (x: a concept or an external object) and an external object $(y)$ to a real number in $[0,1]$ between $\mathrm{x}$ and $\mathrm{y}$.

\subsection{Domain Synthesis:}

An ontology is a compact data structure containing information about concepts and the relationships between them. For a Domain and Curriculum ontology [35], the system measures the similarities between concepts to determine the relationship between them in the form of n pairs from $\left(C_{i}, S_{i}\right)$ by $f_{i}(c, n, s)$, where $\mathrm{c}$ is a concept (ID), $\mathrm{n}$ is a natural number and $\mathrm{s}$ is a real number in $(0,1]$, i.e. $C_{1}, C_{2}, \ldots$ are the concepts of the ontology most similar to $C$ and $s_{i} \geq s$ is the similarity between $C$ and $C_{i}$. In addition, the system returns the set of concepts which may correspond to the term.

\section{Experimental Work:}

An E-learning service can be divided to static, dynamic and hybrid processing. The static processing represents contents of the course and the dynamic processing is introduced through personalized processing and is shown in the hybrid processing. In a practical application of the $I E_{L} S M_{A}$, which depends on dynamic processing, transforms the role of the student from the recipient to deduced, through the student's contribution to learning materials and participation in activities and discussions on learning materials incomprehensible. In the initial part of the system utilization is the user authentication. Each type of users (student - instructor - expert - admin) registered account will possess different access levels and different ontology creation from the knowledge formed in each user's learning level as shown in figure 8 .

\section{Fig. 8: User's GUI}

The $I E_{L} S M_{A}$ design implementation synchronous as shown in figure 9, which includes vision and mission of the system. Next, multi-agent will be used to develop and organize knowledge resources, which can support course content in the e-learning system.

\section{Fig.9: Vision and Mission of the proposed system.}

There are several agents used in the system namely ontology, semantic service and linked data agents.

The ontology agent is used to produce domain and curriculum. Domain Ontology (DO) is not only important for e-learning purposes but for semantic web application, it describes the basic theoretical concepts and relations in the area that is being taught. The DO goal is to track the progress of learners mastering specific domain, the course authors should introduce knowledge using this type of ontologies. DO can be classified into two categories, the first represents the basic concepts of the domain under consideration along with their interrelations and basic properties as shown in figure 10. The second represents hierarchical and navigational relationships, which defines the logical structure of the content that is generally subjective and highly depends on ontology application goals as shown in figure 11. 


\section{A- Hierarchal Layout.}

\section{B- Forced Directed Layout.}

Fig. 10: Layout Types of Domain Ontology.

Fig. 11: Logical Structure of Domain Ontology.

Curriculum Ontology $(\mathrm{CO})$ is complementing the DO by representing the semantic of the problem that has been solved. The content problem types, structures, areas, activities, and steps that learner should follow during the problem-solving process are linked in this ontology that includes the concepts and relations. Additionally, the user personalization is supported in the where users can customize their e-learning interface for both their self-collected resources by searching and system-suggested resources by experts. CO organizes the knowledge resources by means of ontology to represents the knowledge as shown in Figure 12.

Fig. 12: Organize the courses by curriculum ontology.

Through the Semantic Service Agent (SSA), each user can play a different role. A user acts as a requester (learner), formulating a service request to sharing, finding and reusing e-learning materials, which support the learners in their learning process in two ways; local context provision and global context provision. The local context provision (Offline) provides the learner with references to summaries, general information, detailed information, examples, and quizzes. Global context provision is provided through two searching techniques as follows; online search and Deep Learning search. The Online search provides the learner with references to additional resources from the semantic web, which helps to improve the learner background on the topics that they want to learn. While the Deep Learning integrates the available resources in Offline as well as Online as shown in figure 13.

Fig. 13: Different searching techniques to obtain the courses content.

A user can also act as a service provider (instructor), for building a concept-based digital course library where subject domain ontology is used for classification of course content by using learning object metadata together with a well-defined knowledge base as shown in the figure 14.

Fig. 14: Example of course content for a user.

Finally, a user can also act as a broker (expert), maintain the concepts description, evaluate the use of domain ontology in an information retrieval tool and comparing to the simple keyword based retrieval without using ontology as shown in figure 15.

Fig. 15: Example of a user as an expert.

The linked data agent (LDA) is used to collect, codify, organize and arrange the knowledge in a systematic order. It is the most difficult and time-consuming stage of any ontology development process. In this respect, LDA is based on two main steps are required to enable a wide integration of various data. The first step, 
educational service integration which integrates the source of education data and content. The second step, education data integration which facilitates interlinking of learning resources and repositories of learning data.

\section{Results and Discussion:}

In this paper, the system is assessed on the basis of the ontology used by the user in determining its priorities and the data is then collected by the data collection agent. The proposed system was compared to Higher Education Ontology Reference (HERO) [36], which describes several aspects of university domain such as organizational structure, administration, staff, roles, incomes, etc. Hero ontology was used as a reference symbolized by the symbol $\left(O_{\text {Ref }}\right)$ while the symbol of the computed ontology is symbolized by $\left(O_{\text {Com }}\right)$.

Fig. 16: Hierarchal Layout for Reference Higher Education Ontology (HERO) [36].

Precision and Recall measures [37] are used to evaluate the proposed ontology where Precision (P) and Recall (R) are defined as:

$$
\begin{aligned}
& P\left(O_{\text {Ref }}, O_{\text {Com }}\right)=\frac{\left|O_{\text {Com }} \cap O_{\text {Ref }}\right|}{\left|O_{\text {Com }}\right|} \ldots \ldots . . . . .(1) \\
& R\left(O_{\text {Ref }}, O_{\text {Com }}\right)=\frac{\left|O_{\text {Com }} \cap O_{\text {Ref }}\right|}{\left|O_{\text {Ref }}\right|} \ldots \ldots . . . .(2)
\end{aligned}
$$

It is interesting that precision and recall are the inverse of each other:

$$
P\left(O_{\text {Ref }}, O_{\text {Com }}\right)=\frac{\left|O_{\text {Com }} \cap O_{\text {Ref }}\right|}{\left|O_{\text {Com }}\right|}=R\left(O_{\text {Com }}, O_{\text {Ref }}\right)
$$

To summarizing overview and for balancing the precision and recall values, F-measure is used as:

$$
F\left(O_{R e f}, O_{C o m}\right)=\frac{2 \cdot P\left(O_{R e f}, O_{C o m}\right) \cdot R\left(O_{R e f}, O_{C o m}\right)}{P\left(O_{R e f}, O_{\text {Com }}\right)+R\left(O_{R e f}, O_{\text {Com }}\right)}
$$

Precision and Recall are used to evaluating the Lexical term layer and Concept of an ontology hierarchies.

The Lexical precision $\left(L_{P}\right)$ and Lexical recall $\left(L_{R}\right)$ are defined as follows:

$$
L_{P}\left(O_{\text {Com }}, O_{R e f}\right)=\frac{\left|O_{\text {Com }} \cap O_{R e f}\right|}{\left|O_{\text {Com }}\right|}, L_{R}\left(O_{\text {Com }}, O_{R e f}\right)=\frac{\left|O_{\text {Com }} \cap O_{\text {Ref }}\right|}{\left|O_{\text {Ref }}\right|}
$$

$L_{p}$ and $L_{R}$ are reflects how good the learned lexical terms cover the target domain.

The influence of inserting and replacing concepts in a hierarchy is shown in Table 1 . The tables contain the sets for ontologies $O_{R e f}$ and $O_{\text {Com }}$. When comparing the calculated Ontology shown in figure 10 and reference Ontology shown in figure 16 with each other, the get result is $L_{P}\left(O_{\text {Com }}, O_{\text {Ref }}\right)=0.743$ and $L_{R}\left(O_{\text {Com }}, O_{\text {Ref }}\right)=0.794$.

Comparing the two concept hierarchies with each other measures are usually divided into two main kinds namely local and a global. The local measure compares the positions of two concepts while the global measure is compares the whole concept hierarchies.

In the local taxonomic measure, the similarity of two concepts will be computed based on a characteristic extract $(C E)$ from the hierarchy of concept. The local taxonomic precision $\left(\dot{\delta}_{p}^{C E}\right)$ of two concepts $c_{1} \in O_{C}$ and $c_{2} \in O_{R}$ is defined as: 


$$
\dot{i}_{p}^{C E}\left(c_{1}, c_{2}, O_{C}, O_{R}\right)=\frac{\left|C E\left(c_{1}, O_{C}\right) \cap C E\left(c_{2}, O_{R}\right)\right|}{\left|C E\left(c_{1}, O_{C}\right)\right|}
$$

To characterize a concept by its super and sub concepts, the taxonomic overlap measure which also called Semantic of a level concept $\left(S_{l c}\right)$ is used. Let the concept $c \in C$ and the ontology $O, S_{l c}$ is defined as follows:

$$
S_{l c}(c, O)=\left\{c_{i} \mid c_{i} \in C \wedge\left(c_{i} \leq c \vee c \leq c_{i}\right)\right\}
$$

The common semantic of a level concept $\left(C S_{l c}\right)$ excludes all concepts which are not also available in the other ontology's set of concepts:

$$
C S_{l c}\left(c, O_{1}, O_{2}\right)=\left\{c_{i} \mid c_{i} \in C_{1} \cap C_{2} \wedge\left(c_{i} \leq c \vee c \leq C_{i}\right)\right\}
$$

Foe a full comprehensive analysis to be done, the computed ontologies need the measures, $\dot{i}_{p}^{C E_{k c}}$ and $\dot{i}_{R}^{C E_{l c}}$ are better suited for evaluation. This is achieved by using the common semantic of a level concept and by computing the taxonomic precision values only for the common concepts of both ontologies.

$$
\begin{aligned}
& i_{p}^{C E_{k c}}\left(O_{C o m}, O_{R e f}\right)=\frac{1}{\left|C_{C o m} \cap C_{R e f}\right|} \sum_{c \in C_{C o m} \cap C_{\text {Ref }}} i_{p}^{C E}\left(c_{1}, C_{2}, O_{c o m}, O_{R e f}\right) \\
& i_{R}^{C E_{c c}}\left(O_{C o m}, O_{R e f}\right)=i_{p}^{C E}\left(O_{R e f}, O_{C o m}\right)
\end{aligned}
$$

Therefore the taxonomic F-measure is introduced, which is the harmonic mean of the global taxonomic precision and recall.

$$
T F\left(O_{C o m}, O_{R e f}\right)=\frac{2 \cdot T P\left(O_{C o m}, O_{R e f}\right) \cdot T R\left(O_{C o m}, O_{R e f}\right)}{T P\left(O_{\text {Com }}, O_{R e f}\right)+T R\left(O_{C o m}, O_{R e f}\right)}
$$

Symmetric global taxonomic overlap measures can be solely derived from taxonomic F-measures.

$$
i=\frac{T F}{2-T F}
$$

Table 1: Comparison between concepts in the calculated Ontology $\left(O_{C o m}\right)$ and the reference Ontology

$$
\left(O_{\text {Ref }}\right) \text {. }
$$

By comparing ontologies $O_{\text {Ref }}$ and $O_{\text {Com }}$, there are some missing concepts. Although the hierarchy of the remaining concepts remained unchanged, the natural language identifier was changed to concepts.

Table 2: Evaluation of the ontologies without and with a common semantic based measure So this has to be reflected by taxonomy measures which are not influenced by errors on the lexical term layer. The criterion of good evaluation measures was that a gradual increase in the error rate should lead to a more or less gradual decrease in the evaluation results. Table 2 shows that $i_{p}^{C E_{k c}}$ and $i_{R}^{C E_{l c}}$ fulfill this criterion. For the calculated ontology with common semantic of a level concept, the results were very convincing while the results in the case of the local taxonomic measure were fairly good as the measure was affected by some errors in lexical term layer as well as errors in the hierarchy of concepts. In addition, this 
measure is more affected by errors in the lexical term layer than the lexical precision and recall measure itself.

\section{Conclusion:}

The progress of peoples is measured by the progress of education, as the world is constantly developing education. One of the most important features that contribute to the development of education, is information technology. E-learning is one of the solutions that help in this development. As a result of the revolution of information, e-learning has been supported by artificial intelligence techniques to raise its efficiency and maximize it.

This paper presents a proposed intelligent e-learning system to manage the e-learning process, based on multi-agent which are introduced by the Semantic Services Agent, the Linked Data Agent, and the Ontology Agent. When evaluating the results, it was found that:

* Both the instructor and the learner were supported by a semantic service agent that helps in the search within the system (Offline) or outside the system (Online) or the integration between the two systems (Deep Learning). All searching types allow users to store queries and be informed, if new learning objects, matching user's criteria, will be added to the system.

* The system contains the Ontology agent, which describes the selected sites, to provide search results accurately and as close as possible to the expectations of the user.

* Integration of the linked data agent to the system to facilitate system management and build an architectural structure for search products that will help improve the learning outcomes.

\section{Reference:}

1. Alexander, B., Barrett, K., Cumming, S., Herron, P., Holland, C., Keane, K, \& Tsao, J. (2016). Report from the Information Overload and Underload Workgroup. Open Scholarship Initiative Proceedings.

2. Frion, P., \& Yzquierdo-Hombrecher, J. (2009, June). How to implement competitive intelligence in SMEs. In Proceedings of the Third VISIO Conference (pp. 162-173).

3. Ashton, D., \& Couzins, M. (2015). Content Curators as Cultural Intermediaries: "My reputation as a curator is based on what I curate, right?". M/C Journal, 18(4).

4. Yang, C. C., Chen, H., \& Hong, K. (2003). Visualization of large category map for Internet browsing. Decision support systems, 35(1), 89-102.

5. Eppler, M. J., \& Mengis, J. (2004). The concept of information overload: A review of literature from organization science, accounting, marketing, MIS, and related disciplines. The information society, 20(5), 325-344.

6. Farhoomand, A. F., \& Drury, D. H. (2002). Overload. Communications of the ACM, 45(10), 127.

7. Henderson, J. C., \& Venkatraman, H. (1999). Strategic alignment: Leveraging information technology for transforming organizations. IBM systems journal, 38(2.3), 472-484.

8. Murdoch, T. B., \& Detsky, A. S. (2013). The inevitable application of big data to health care. Jama, 309(13), 1351-1352.

9. Lehmann, J., Isele, R., Jakob, M., Jentzsch, A., Kontokostas, D., Mendes, P. N., \& Bizer, C. (2015). DBpedia-a large-scale, multilingual knowledge base extracted from Wikipedia. Semantic Web, 6(2), 167-195.

10. Morton, T. (2013). Realist magic: Objects, ontology, causality. Open Humanities Press. 
11. Rodríguez, N. D., Cuéllar, M. P., Lilius, J., \& Calvo-Flores, M. D. (2014). A fuzzy ontology for semantic modelling and recognition of human behaviour. Knowledge-Based Systems, 66, 46-60.

12. Bizer, C., Heath, T., \& Berners-Lee, T. (2011). Linked data: The story so far. In Semantic services, interoperability and web applications: emerging concepts (pp. 205-227). IGI Global.

13. Cambria, E., \& White, B. (2014). Jumping NLP curves: A review of natural language processing research. IEEE Computational intelligence magazine, 9(2), 48-57.

14. Rani, M., Nayak, R., \& Vyas, O. P. (2015). An ontology-based adaptive personalized e-learning system, assisted by software agents on cloud storage. Knowledge-Based Systems, 90, 33-48.

15. Kiljander, J., D’elia, A., Morandi, F., Hyttinen, P., Takalo-Mattila, J., Ylisaukko-Oja, A., \& Cinotti, T. S. (2014). Semantic interoperability architecture for pervasive computing and internet of things. IEEE access, 2, 856-873.

16. MacLennan, E., \& Van Belle, J. P. (2014). Factors affecting the organizational adoption of serviceoriented architecture (SOA). Information Systems and e-Business Management, 12(1), 71-100.

17. Deng, L., Wu, J., \& Hu, Z. (2008, May). ISCF: A Semantic Web Service Composition Framework Based on OAA. In 2008 The 3rd International Conference on Grid and Pervasive ComputingWorkshops (pp. 250-255). IEEE.

18. Burstein, M., Bussler, C., Finin, T., Huhns, M. N., Paolucci, M., Sheth, A. P., \& Zaremba, M. (2005). A semantic web services architecture. IEEE Internet Computing, 9(5), 72-81.

19. Madhu, G., Govardhan, D. A., \& Rajinikanth, D. T. (2011). Intelligent semantic web search engines: a brief survey. arXiv preprint arXiv:1102.0831.

20. Ouni, A., Kessentini, M., Inoue, K., \& Cinnéide, M. O. (2015). Search-based web service antipatterns detection. IEEE Transactions on Services Computing, 10(4), 603-617.

21. Bettini, C., Brdiczka, O., Henricksen, K., Indulska, J., Nicklas, D., Ranganathan, A., \& Riboni, D. (2010). A survey of context modelling and reasoning techniques. Pervasive and Mobile Computing, 6(2), 161-180.

22. Fan, James, et al. "Automatic knowledge extraction from documents." IBM Journal of Research and Development 56.3 .4 (2012): 5-1.

23. Jain, V., \& Singh, M. (2013). Ontology development and query retrieval using protégé tool. International Journal of Intelligent Systems and Applications (IJISA), 5(9), 67.

24. Jones, D., Bench-Capon, T., \& Visser, P. (1998). Methodologies for ontology development.

25. Rose, G. (2016). Visual methodologies: An introduction to researching with visual materials. sage.

26. Burov, E. (2014). Complex ontology management using task models. International Journal of Knowledge-Based and Intelligent Engineering Systems, 18(2), 111-120.

27. Bizer, C., Heath, T., \& Berners-Lee, T. (2011). Linked data: The story so far. In Semantic services, interoperability and web applications: emerging concepts (pp. 205-227). IGI Global.

28. Heitmann, B., \& Hayes, C. (2010, March). Using linked data to build open, collaborative recommender systems. In 2010 AAAI Spring Symposium Series.

29. Stauber, C., Ambrose, J., \& Rothwein, T. M. (2003). U.S. Patent No. 6,574,635. Washington, DC: U.S. Patent and Trademark Office.

30. Bechhofer, S., van Harmelen, F., Hendler, J., Horrocks, I., McGuinness, D. L., Patel-Schneider, P. F., ... \& Schreiber, G. (2006). OWL Web Ontology Language: Reference. W3C, 2004.

31. Willett, P. (2006). The Porter stemming algorithm: then and now. Program, 40(3), 219-223. 
32. Robertson, S. (2004). Understanding inverse document frequency: on theoretical arguments for IDF. Journal of documentation, 60(5), 503-520.

33. Nicol, G., Wood, L., Champion, M., \& Byrne, S. (2001). Document object model (DOM) level 3 core specification. W3C Working Draft, 13, 1-146.

34. Fionda, V., \& Pirrò, G. (2018, July). Fact checking via evidence patterns. In Proceedings of the 27th International Joint Conference on Artificial Intelligence (pp. 3755-3761). AAAI Press.

35. Gašević, D., \& Hatala, M. (2006). Ontology mappings to improve learning resource search. British Journal of Educational Technology, 37(3), 375-389.

36. Zemmouchi-Ghomari, L., \& Ghomari, A. R. (2013). Translating natural language competency questions into SPARQLQueries: a case study. In The First International Conference on Building and Exploring Web Based Environments (pp. 81-86).

37.Euzenat, J. (2007, January). Semantic Precision and Recall for Ontology Alignment Evaluation. In IJCAI (Vol. 7, pp. 348-353). 